\title{
Influência da superabundância por Aulonemia aristulata (Bambuseae) sobre o banco de sementes transitório em um fragmento de Floresta Atlântica ${ }^{1}$
}

\author{
Influence of Aulonemia aristulata (Bambuseae) overabundance \\ on the transient seed bank of an Atlantic Forest fragment
}

\author{
Daniella Vinha ${ }^{1,5}$, Luciana Ferreira Alves ${ }^{4}$, Lilian Beatriz Penteado Zaidan ${ }^{3}$ \\ \& Maria Tereza Grombone-Guaratini ${ }^{2}$
}

\begin{abstract}
Resumo
Este estudo avaliou a estrutura e composição de espécies no banco de sementes transitório em duas áreas de mata atlântica do Parque Estadual Fontes do Ipiranga: uma área onde o bambu nativo Aulonemia aristulata é superabundante e outra área sem bambus, ambas localizadas em um fragmento de floresta secundária do Domínio da Mata Atlântica. Nossa hipótese foi a de que o banco de sementes transitório da área onde o bambu é superabundante possui menor número de sementes e diminuição da riqueza de espécies comparada à área sem bambu. Se confirmada a hipótese, sugerimos que isso deve limitar o processo de regeneração florestal por meio do banco de sementes transitório, o que deve contribuir para a perpetuação do bambu e para a manutenção do estado de distúrbio. Coletamos, em cada área, 45 amostras de serapilheira e separamos, quantificamos e identificamos as sementes no menor nível taxonômico possível. Na área de superabundância de bambu encontramos menor riqueza de espécies e maior proporção de espécies exclusivas. Entretanto, não encontramos diferenças na abundância de sementes no banco transitório entre as duas áreas. Nossos resultados mostraram que a distribuição espacial de sementes na área onde o bambu é superabundante foi mais limitada que na área sem bambu uma vez que cerca de 50\% das amostras coletadas na área com bambu não apresentaram nenhuma semente. Não foi possível comprovar se a redução na riqueza de espécies foi consequência da menor densidade de espécies arbóreas na área ou efeito da superabundância de bambus. A diminuição da riqueza de espécies e a limitação espacial no banco de sementes transitório encontrada na área onde o bambu é dominante sugerem a diminuição da contribuição desta via de regeneração da vegetação.
\end{abstract}

Palavras-chave: dispersão de sementes, fragmentação florestal, regeneração natural.

\begin{abstract}
The aim of our study was to evaluate the community structure and species composition of seeds present in the transient seed bank in two areas of Atlantic forest fragment in São Paulo: an area where the bamboo is overabundant and another without bamboos, both located in a secondary forest fragment. Our hypothesis is that bamboo overabundance influences the availability and species diversity of seeds in the transient soil seed bank, what, in turn, can influence the potential for natural forest recovery. We collected 45 samples of litter in each site and sorted, quantified and identified seeds to the most precise taxonomic levels. We found lower species richness and higher proportion of exclusive species in the area where bamboo is overabundant, but there was no difference in the abundance of seeds in the transient seed bank between areas. Our results show that seed distribution in the bamboo overabundant area was more limited than in control area because $50 \%$ of collected samples did not present any seeds. It was not possible to conclude whether the reduction on species richness of the transient seed bank was a result of the lower density of tree species or just a side effect of the overabundance of bamboo. The decrease in species richness and seed dispersal limitation in transient seed bank found in bamboo overabundant area suggest the decreased contribution of this pathway regeneration of vegetation. Key words: seed dispersal, forest fragmentation, natural regeneration.
\end{abstract}

\footnotetext{
${ }^{1}$ Parte da dissertação de Mestrado da primeira autora pelo Prog. Pós-graduação em Biodiversidade Vegetal e Meio Ambiente do Instituto de Botânica, São Paulo, SP, Brasil ${ }^{2}$ Instituto de Botânica de São Paulo, Núcleo de Ecologia, Av. Miguel Stéfano 3687, Água Funda, 04301-902, São Paulo, SP, Brasil.

${ }^{3}$ Instituto de Botânica de São Paulo, Núcleo de Pesquisa em Fisiologia e Bioquímica de Plantas, Av. Miguel Stéfano 3687, Água Funda, 04301-902, São Paulo, SP, Brasil.

${ }^{4}$ University of California, Inst. of the Environment and Sustainability, 619 Charles E. Young Dr. East, La Kretz Hall, Suite 300, 90095, Los Angeles, CA, USA.

${ }^{5}$ Autor para correspondência: dvinha@gmail.com
} 


\section{Introdução}

Perturbações antrópicas representam uma das fontes mais intensas de alterações na estrutura e composição de espécies em florestas tropicais (Shackelford et al. 2013). Embora bambus sejam descritos como parte importante da diversidade florestal na América do Sul (Filgueiras \& Pereira 1998) tais espécies podem proliferar substancialmente após perturbações antrópicas e se tornar superabundantes (Garrot et al. 1993; Lima et al. 2012). A vigorosa capacidade de crescimento e produção de biomassa dos bambus nativos da Mata Atlântica (Narukawa \& Yamamoto 2002), sua superioridade competitiva (Griscom \& Ashton 2003, 2006) e a plasticidade de traços funcionais como o ajuste das taxas fotossintéticas (Mulkey 1986; Gratani et al. 2008; Montti et al. 2013) podem explicar o sucesso e, portanto, a superabundância dos bambus em áreas perturbadas (Montti et al. 2013; Grombone-Guaratini et al. 2014).

Vários estudos demonstram que a superabundância de bambus têm um forte efeito sobre a estrutura e composição das florestas tropicais, diminuindo a densidade de árvores, área basal total, riqueza florística e diversidade de grupos funcionais de plantas (Campanello et al. 2007; González et al. 2002; Griscom \& Ashton 2003; 2006; Griscon et al. 2007; Larpken et al. 2011; Montti et al. 2013; d'Oliveira et al. 2013) além de propiciar alterações na riqueza e no padrão de distribuição da chuva de sementes (Rother et al. 2009, 2015, 2016).

Bambus lenhosos são espécies monocárpicas que florescem, no Brasil, em média a cada 30-40 anos (Filgueiras \& Santos-Gonçalves 2004). Em situações onde espécies de bambu atingem superabundância, o estado de perturbação das florestas pode perdurar por longos períodos, em função da baixa capacidade de resiliência da comunidade arbórea e de determinados atributos da história de vida de bambus, como hábito, caracteres reprodutivos, longevidade e superioridade competitiva. Isso ocorre porque as vias de regeneração natural, como a chuva de sementes, banco de sementes e o banco de plântulas, são influenciadas pelos níveis de perturbação da floresta, necessitando de planos de manejo e recuperação direcionados (Alvarez-Aquino et al. 2005; Griscom \& Ashton 2006). Esses efeitos são exemplificados por alguns estudos em áreas com dominância por bambu que têm demonstrado a inibição do estabelecimento de espécies arbóreas, o aumento na mortalidade de plântulas, alterações na densidade e composição do banco de sementes do solo e mudanças na composição florística e funcional da chuva de sementes (Widmer 1998; Griscom \& Ashton 2003; Rother et al. 2009; Vinha et al. 2011; Grombone-Guaratini et al. 2014).

Sabe-se que o banco de sementes do solo pode afetar a estrutura e composição da vegetação após perturbação, sendo considerado efetivo para a regeneração de áreas degradadas ou facilitando o restabelecimento da vegetação após o manejo (Omand et al. 2014). Contudo, avaliações consistentes do potencial de regeneração natural em florestas tropicais demandam longos períodos de tempo (em geral, mais de um ano de monitoramento da disponibilidade de sementes e composição de espécies da chuva de sementes) e estrutura física para acompanhamento de experimentos (e.g., a avaliação do banco de sementes em casas de vegetação). Uma alternativa possível consiste na avaliação da estrutura e composição de espécies de sementes contidas na serapilheira. Tais sementes são consideradas integrantes do banco de sementes transitório (sensu Garwood 1989) que é composto por sementes grandes (maiores que $5 \mathrm{~mm}$ ), o que diminui a probabilidade de incorporação ao solo (Foster 1986; Farrell et al. 2012). Alguns estudos descreveram o uso potencial da serapilheira em técnicas de transposição para recuperação de áreas degradadas (Souza et al. 2006; Rodrigues et al. 2010; Mônico 2012), mas informações sobre a estrutura e composição desse componente da regeneração natural em áreas com dominância por bambus são inexistentes no Brasil. Dada a importância da regeneração natural em florestas tropicais (Alvarez-Buylla \& García Barrios 1991; Dalling \& Hubbell 2002; Castillo \& Stevenson 2010) é de fundamental importância avaliar a influência que os bambus exercem sobre a estrutura da vegetação e do banco de sementes.

O objetivo desse estudo foi comparar a estrutura (número de propágulos, densidade, frequência) e composição de espécies do banco de sementes transitório em uma área onde o bambu é superabundante em relação à outra área próxima sem a presença de bambu em um fragmento de floresta secundária Atlântica no Brasil. Especificamente, estudamos o efeito da superabundância do bambu sobre a disponibilidade de sementes do banco de sementes transitório e, consequentemente, sobre o potencial de regeneração da floresta. Testamos a hipótese de que o banco de sementes transitório da área em que o 
bambu é superabundante apresenta menor número de sementes e diminuição da riqueza de espécies. A confirmação da hipótese sugere a limitação do processo de regeneração florestal por meio do banco de sementes transitório, o que deve contribuir para a perpetuação do bambu e para a manutenção do estado de distúrbio. Por outro lado, a refutação da hipótese sugere que esta via de regeneração natural não é afetada pela superabundância de bambus, podendo ser um importante processo pelo qual a regeneração natural encontra suporte.

\section{Material e Métodos}

Área de estudos

$\mathrm{O}$ estudo foi realizado no Parque Estadual das Fontes do Ipiranga (PEFI), localizado na região sudeste do Município de São Paulo, SP (2338'40'S, 46³6'38''W; 770-825 m de altitude; Nastri et al. 1992) com solo do tipo Latossolo Vermelho-Amarelo Distrófico (EMBRAPA 2006). O clima da região classifica-se como Cwb, i.e., tropical temperado (Köppen 1948). A precipitação média anual é de $1540 \mathrm{~mm}$, as temperaturas médias mensais máximas ocorrem no mês de fevereiro $\left(22,4^{\circ} \mathrm{C}\right)$ e as mínimas em julho $\left(15,7^{\circ} \mathrm{C}\right)$ (Santos \& Funari 2002). O PEFI tem 526,38 ha de área total, dos quais $63,5 \%$ ou 350 ha (Pivelo \& Peccinini 2002) são representados por Floresta Ombrófila Densa Montana (sensu Oliveira-Filho \& Fontes 2000). A vegetação é composta por floresta secundária, com algumas áreas muito perturbadas, onde espécies pioneiras estão presentes na vegetação nos estádios iniciais de sucessão ecológica (Pivello \& Peccinini 2002). Aulonemia aristulata (Döll) MacClure (Poaceae: Bambusoideae) é uma espécie de bambu lenhoso, nativo da Mata Atlântica, que ocorre na região Central e no Sudeste do Brasil em baixas densidades populacionais. No PEFI, essa espécie de bambu ocorre em densidades muito baixas em áreas de estádio sucessional avançado ou médio (Shirasuna \& Filgueiras 2013) e prevalece com densidades elevadas em bordas de florestas e áreas perturbadas, cujos colmos crescem até $4 \mathrm{~m}$ de altura apoiando-se na vegetação (Clark 2001).

Para a realização deste estudo amostramos duas áreas dentro do fragmento do PEFI, as quais são distantes entre si cerca de 1,5 km: uma área degradada com a presença de espécies invasoras, onde o bambu Aulonemia aristulata é superabundante (área CB - ou "com bambu", coordenadas 2339'79'S, 46³7'20'W), a qual foi comparada com uma área pouco perturbada do
PEFI (área SB - ou "sem bambu", coordenadas 2338'84'S, 46³7'03'W), com dossel fechado e sem dominância de espécies invasoras (Fig. 1). Em um levantamento prévio realizado nas áreas deste estudo, Vinha et al. (2011) verificaram que a densidade total de árvores (diâmetro à altura do peito $\geq 4,8$ centímetros) foi menor em CB (1.354 caules $/$ ha $^{-1}$ ) em comparação com a área SB (1.767 caules $\left./ \mathrm{ha}^{-1}\right)$. Além disso, aqueles autores mostraram também que a média de abertura de dossel ( $\%$ média \pm IC 95\%) foi maior na área CB (12,05 \pm $0,42)$ do que em SB $(8,10 \pm 0,36)$. O mesmo padrão foi observado para a radiação incidente difusa (CB $=21,96 \pm 0,66 ; \mathrm{SB}=11,65 \pm 0,51)$.

\section{Levantamento de campo}

Em cada uma das duas áreas, estabelecemos uma parcela de $50 \times 140 \mathrm{~m}(0,7 \mathrm{ha})$ dividida em sub-parcelas $(10 \times 10 \mathrm{~m})$, totalizando 70 unidades amostrais. Em outubro de 2006, 45 amostras de serapilheira foram coletadas aleatoriamente em cada uma das áreas. As amostras consistiram na camada de material vegetal localizada imediatamente acima do solo, o que caracteriza o banco de sementes transitório (Simpson et al. 1989). O período de coleta correspondeu ao pico de dispersão na chuva de sementes observado nessas áreas (Penhalber \& Mantovani 1997). As coletas foram feitas usando um quadrado de madeira de 20 $\times 20 \mathrm{~cm}$, os quais consistiram em nossas unidades amostrais, totalizando uma área amostral de 1,8 $\mathrm{m}^{2}$ em cada área estudada. As sementes foram contadas e classificadas em morfoespécies e as espécies identificadas por especialistas. A classificação das espécies em famílias seguiu a proposta de APG IV (2016).

Caracterizamos a estrutura do banco de sementes transitório em termos de abundância total de sementes, riqueza de espécies, e densidade e frequência relativa de sementes por espécie. Nós comparamos a densidade total de sementes da serapilheira entre as áreas através do teste não paramétrico de Mann-Whitney (Zar 2010), uma vez que os dados não tiveram distribuição normal e não apresentaram homogeneidade de variâncias, mesmo após transformação em escala log +1 e raiz quadrada. Fixamos o nível de significância em $p<0.05$. Para a realização das análises usamos o software SigmaStat 3.5, SPSS Inc., USA. Calculamos a densidade e a frequência absoluta e relativa para cada morfoespécie buscando avaliar o padrão de distribuição das sementes nas áreas amostrais (Müeller-Dombois \& Ellenberg 1974). 

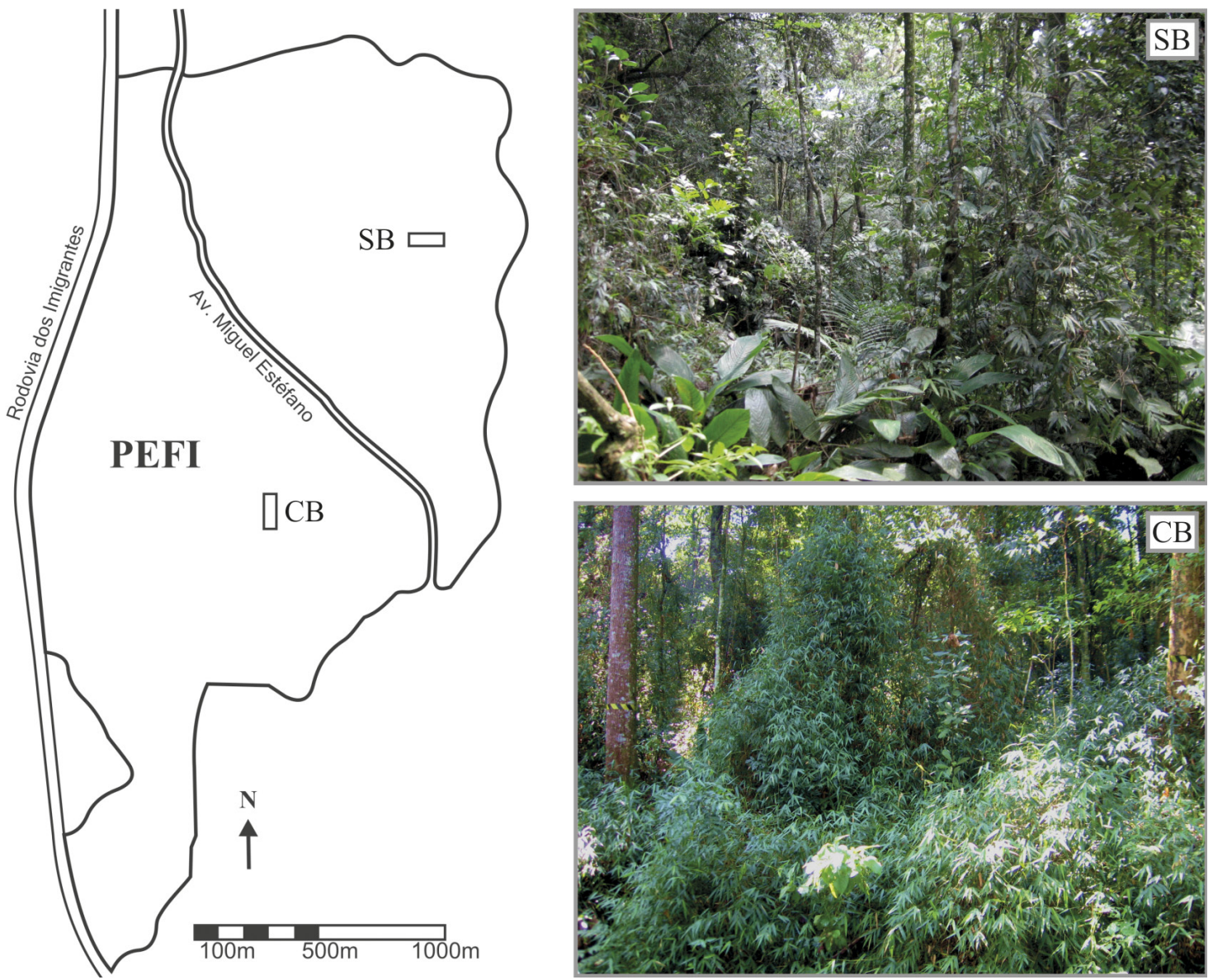

Figura 1 - Localização e aspecto geral da vegetação das áreas com superabundância por bambu (CB) e sem bambu (SB) no Parque Estadual das Fontes do Ipiranga (PEFI), São Paulo, SP.

Figure 1 - Location and general appearance of the vegetation of the bamboo overabundant-area $(\mathrm{CB})$ and without bamboo $(\mathrm{SB})$ in the Parque Estadual das Fontes do Ipiranga (PEFI), São Paulo, SP, Brazil.

Comparamos a composição de espécies entre as parcelas amostrais de ambas as áreas com o índice de similaridade Bray-Curtis (Sørensen quantitativo), considerando a abundância das espécies (Magurran \& Mcgill 2011). Também utilizamos uma análise de rarefação para compararmos a riqueza de espécies entre as áreas, visando evitar subestimativas devido a diferenças na abundância de sementes em cada uma delas (Gotelli \& Colwell 2001). Para esta análise, a riqueza esperada foi calculada para um mesmo número de sementes entre as amostras das duas áreas (100, 170 e 240 sementes), com um intervalo de confiança estabelecido a partir de 1000 reamostragens, com base no número total de espécies verificadas nas duas áreas $(n=40)$ (como em Vinha et al. 2011). Para esses cálculos, utilizamos o programa EcoSim 7.0 (Gotelli \& Entsminger 2001).

\section{Resultados}

No total foram amostradas 539 sementes na serapilheira das duas áreas de coleta, as quais foram discriminadas em 40 espécies ou morfoespécies distribuídas em 22 gêneros e 15 famílias. Na área CB foram amostradas 296 sementes e 17 espécies, enquanto na área SB foram amostradas 243 sementes e 29 espécies. Nenhuma semente foi identificada como exótica naturalizada ou invasora (ver Moro et al. 2012) no banco transitório (Tab. 1). Arecaceae, Asteraceae, Meliaceae e Sapindaceae contribuíram com $47 \%$ da riqueza de espécies e $66 \%$ da abundância de sementes na área $\mathrm{CB}$, enquanto Asteraceae e Myrtaceae foram as principais famílias encontradas na área $\mathrm{SB}$, contribuindo com $38 \%$ da riqueza de espécies e $77 \%$ da abundância total desta área. Mikania 
Tabela 1 - Lista de espécies contidas nas amostras de serapilheira nas áreas com superabundância por bambu (CB) e sem bambu (SB) no Parque Estadual das Fontes do Ipiranga, São Paulo, Brasil. As espécies foram classificadas segundo formas de vida (FV) em árvore/arbusto (Arv/Arb), trepadeira (Trep) e palmeira (Palm). O número de sementes (N), a densidade (DR) e frequência relativa (FR) para cada espécie são apresentados. A nomenclatura segue o APG III (2009). Table 1 - List of species contained in the litter samples of bamboo-dominated area (CB) and control area (SB) in the Parque Estadual das Fontes do Ipiranga, São Paulo, Brazil. Species were sorted according to growth form (FV) in tree/shrub (Arv/Arb), vine (Trep) and palm (Palm). Number of seeds (N) and relative density (DR) and frequency (FR) are provided for each species. Nomenclature follows APG III (2009).

\begin{tabular}{llllll}
\hline Famílias / Espécies & FV & N & DR & FR
\end{tabular}

Área com Bambu (CB)

Arecaceae

Geonoma cf. schottiana Mart.

Syagrus romanzoffiana (Cham.) Glassman

Asteraceae

Mikania cordifolia (L.F.) Willd.

Vernonia difusa Less.

Fabaceae

Piptadenia gonoacantha (Mart.) J.F. Macbr.

Meliaceae

Guarea sp.

Trichilia sp.

Myrtaceae

Myrtaceae sp.1

Phytolaccaceae

Seguieria langsdorffii Moq.

Primulaceae

Myrsine sp.

Rosaceae

Prunus sp.

Sapindaceae

Allophylus sp.

Cupania cf. oblongifolia Mart.

Indeterminadas

Indet. \#1

Indet. \#2

Indet. \#3

Indet. \#6

$\begin{array}{llll}\text { Palm } & 4 & 1,35 & 2,38 \\ \text { Palm } & 3 & 1,01 & 7,14\end{array}$

Trep

138

46,62

21,43

Arv/Arb

31

10,47

7,14

Arv/Arb

1

0,34

Arv/Arb

1

0,34

2,38

Arv/Arb

2

0,68

2,38

Arv/Arb

20

6,76

11,90

Arv/Arb

4

1,35

4,76

Arv/Arb

57

19,26

Arv/Arb

6

2,03

4,76

Arv/Arb

2

0,68

2,38

Arv/Arb

15

5,07

16,67

-

3

1,01

4,76$$
-
$$

0,34

2,38

7

2,36

1

0,34

2,38

\section{Área sem Bambu (SB)}

Arecaceae

Euterpe edulis Mart.

Syagrus romanzoffiana (Cham.) Glassman

Palm

1

0,41

2,17

Palm

7

2,88

8,70 


\begin{tabular}{|c|c|c|c|c|}
\hline Famílias / Espécies & FV & $\mathbf{N}$ & DR & FR \\
\hline \multicolumn{5}{|l|}{ Apocynaceae } \\
\hline Asclepiadoideae & - & 1 & 0,41 & 2,17 \\
\hline \multicolumn{5}{|l|}{ Asteraceae } \\
\hline Asteraceae sp. & - & 3 & 1,23 & 2,17 \\
\hline Eupatorium sp. & Arv/Arb & 1 & 0,41 & 2,17 \\
\hline Mikania cordifolia (L.F.) Willd. & Trep & 153 & 62,96 & 13,0 \\
\hline Vernonia difusa Less. & Arv/Arb & 4 & 1,65 & 2,17 \\
\hline \multicolumn{5}{|l|}{ Elaeocarpaceae } \\
\hline Sloanea monosperma Vell. & Arv/Arb & 1 & 0,41 & 2,17 \\
\hline \multicolumn{5}{|l|}{ Fabaceae } \\
\hline Machaerium sp. & - & 2 & 0,82 & 4,35 \\
\hline Piptadenia gonoacantha (Mart.) J.F. Macbr. & Arv/Arb & 2 & 0,82 & 4,35 \\
\hline \multicolumn{5}{|l|}{ Lauraceae } \\
\hline Cryptocarya sp. & Arv/Arb & 6 & 2,47 & 8,70 \\
\hline \multicolumn{5}{|l|}{ Malvaceae } \\
\hline Triumfetta semitriloba Jacq. & Arv/Arb & 1 & 0,41 & 2,17 \\
\hline \multicolumn{5}{|l|}{ Meliaceae } \\
\hline Guarea sp. & Arv/Arb & 3 & 1,23 & 2,17 \\
\hline Trichilia sp. & Arv/Arb & 6 & 2,47 & 2,17 \\
\hline \multicolumn{5}{|l|}{ Myrtaceae } \\
\hline Myrcia cf. fallax (Rich.) DC. & Arv/Arb & 1 & 0,41 & 2,17 \\
\hline Myrcia sp. & Arv/Arb & 7 & 2,88 & 2,17 \\
\hline Myrtaceae sp.1 & Arv/Arb & 1 & 0,41 & 2,17 \\
\hline Myrtaceae sp.2 & Arv/Arb & 1 & 0,41 & 2,17 \\
\hline Myrtaceae sp.3 & Arv/Arb & 6 & 2,47 & 2,17 \\
\hline Myrtaceae sp. 4 & Arv/Arb & 1 & 0,41 & 2,17 \\
\hline Myrtaceae sp.5 & Arv/Arb & 9 & 3,70 & 2,17 \\
\hline \multicolumn{5}{|l|}{ Rubiaceae } \\
\hline Amaioua sp. & Arv/Arb & 3 & 1,23 & 2,17 \\
\hline \multicolumn{5}{|l|}{ Sapindaceae } \\
\hline Cupania cf. vernalis Cambess. & Arv/Arb & 8 & 3,29 & 8,70 \\
\hline \multicolumn{5}{|l|}{ Solanaceae } \\
\hline Solanaceae sp.1 & - & 1 & 0,41 & 2,17 \\
\hline \multicolumn{5}{|l|}{ Indeterminadas } \\
\hline Indet. \#1 & - & 6 & 2,47 & 4,35 \\
\hline Indet. \#4 & - & 1 & 0,41 & 2,17 \\
\hline Indet. \#5 & - & 4 & 1,65 & 2,17 \\
\hline Indet. \#7 & - & 2 & 0,82 & 2,17 \\
\hline Indet. \#8 & - & 1 & 0,41 & 2,17 \\
\hline
\end{tabular}




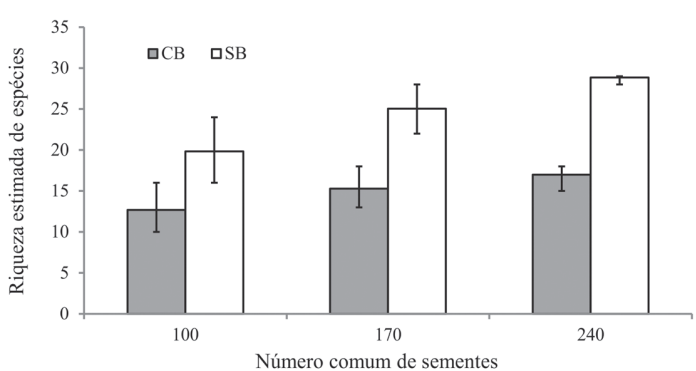

Figura 2 - Riqueza estimada de espécies (média \pm intervalo de confiança a 95\%) para todas as espécies amostradas no banco de sementes transitório em áreas com superabundância por bambu (CB) e sem bambu (SB) no Parque Estadual das Fontes do Ipiranga, São Paulo, Brasil. As estimativas consideraram números comuns de indivíduos $(100,170$ e 240) entre as áreas. Figure 2 - Estimated species richness (mean $\pm 95 \%$ confidence intervals) for all species sampled in the soil seed bank in the bamboo-dominated area (CB) and in the area without bamboo $(\mathrm{SB})$ in the Parque Estadual das Fontes do Ipiranga, São Paulo, Brazil. The estimates considered the number of individuals common $(100,170$ and 240$)$ between areas.

cordifolia (L.F.) Willd. (Asteraceae) foi a espécie mais abundante nas duas áreas, compreendendo entre $46 \%(\mathrm{CB})$ e $62 \%(\mathrm{SB})$ do total de sementes (Tab. 1). Por outro lado, espécies da família Myrtaceae foram mais ricas na área SB $(24,1 \%$ do total de espécies) em comparação com a área CB (5,5\%).

Comparativamente, houve alta proporção de espécies exclusivas em ambas as áreas (75,8 e $61,1 \%$ para as áreas SB e CB, respectivamente), o que resultou em baixa similaridade florística entre elas $($ Sørensen $=0,30)$. A riqueza média estimada foi significativamente maior na área $\mathrm{SB}$ em comparação com a área $\mathrm{CB}$ em todas as simulações (Fig. 2). Não encontramos diferenças significativas na densidade de sementes entre as áreas $(6,6$ sementes. $\mathrm{m}^{-2}$ na área $\mathrm{CB}$ e 5,4 sementes. $\mathrm{m}^{-2}$ na área $\mathrm{SB}, \mathrm{U}=933,5, \mathrm{p}=0,51)$. Entretanto, na área $\mathrm{CB}, 48 \%$ das amostras não continham sementes, sendo esse percentual inferior na área SB (31\% das amostras).

\section{Discussão}

Nosso estudo mostrou que a área onde o bambu é superabundante (CB) apresentou uma expressiva redução na riqueza de espécies. Apesar da abundância de sementes não diferir entre as áreas, encontramos um maior número de amostras sem sementes na área com bambu sugerindo limitação espacial na distribuição de sementes. Desse modo, os resultados suportam parcialmente a nossa hipótese inicial de que a superabundância de Aulonemia aristulata influencia negativamente a riqueza de espécies e a disponibilidade de sementes para as etapas posteriores da regeneração florestal.

Estudos avaliando outros processos do ciclo de regeneração nas mesmas áreas em que este estudo foi realizado mostraram que na área $\mathrm{CB}$ houve menor riqueza de espécies na chuva de sementes (Grombone-Guaratini et al. 2014) e, contrariamente, maior riqueza de espécies no banco de sementes do solo (Vinha et al. 2011). Comparativamente, muitas espécies verificadas na chuva de sementes de ambas as áreas foram coincidentes com aquelas encontradas no banco transitório, tais como Mikania cordifolia (L.F.) Willd., Piptocarpha oblonga (Gardn.) Baker, Vernonia diffusa Less., Syagrus romanzoffiana (Cham.) Glassman, Seguieria langsdorffii Moq., Prunus sp. e Cupania spp., entre outras. Esses resultados sugerem a influência direta da chuva de sementes no banco transitório, uma vez que as sementes contidas na serapilheira resultam de eventos mais recentes de dispersão (Garwood 1989). Consequentemente, a riqueza de espécies do banco de sementes transitório tende a ser semelhante aquela verificada na chuva de sementes do local. Isso mostra que a superabundância do bambu, com suas consequências na estrutura florestal, como a redução da densidade arbórea e a alteração dos rumos do processo sucessional, possivelmente interfere no número de espécies disponíveis para a regeneração natural tanto nas etapas da chuva de sementes (Grombone-Guaratini et al. 2014) quanto na formação do banco de sementes transitório. Por outro lado, os resultados do banco de sementes transitório obtidos por este estudo em relação aos do banco de sementes permanente obtidos por Vinha et al. (2011) corroboram o que foi previamente demonstrado por outros autores, que estes dois componentes do banco de sementes diferem no conteúdo de espécies e, portanto, na efetiva contribuição do processo de regeneração florestal.

Famílias representativas da Mata Atlântica e da vegetação do PEFI, como Arecaceae, Meliaceae e Sapindaceae (Tabarelli \& Mantovani 1999; Barros et al. 2002) foram bem representadas no banco de sementes transitório, sugerindo sua participação na dinâmica da regeneração das áreas. Entretanto, a ocorrência reduzida de espécies típicas de estádios mais tardios de sucessão, como 
as da família Myrtaceae, e a grande proporção de espécies exclusivas sugerem que a dominância por bambu pode alterar a composição de espécies do banco transitório.

Embora não tenhamos encontrado diferença na abundância de sementes do banco transitório entre as áreas, cerca de $50 \%$ das unidades amostrais na área $\mathrm{CB}$ não continham sementes, representando uma diferença 17\% maior em relação à área $\mathrm{SB}$. Isso sugere que o estado de perturbação florestal pode influenciar negativamente a disponibilidade espacial de sementes durante essa fase do ciclo de regeneração. Estudos realizados em outras áreas de Mata Atlântica têm demonstrado a existência de padrões espaciais agregados na chuva de sementes e no estrato de plântulas (Rother et al. 2015), além de maior limitação de sementes em áreas com dominância por bambus (Rother et al. 2009). Uma vez que o banco de sementes transitório representa uma fase de transição entre a chuva de sementes e o recrutamento de plântulas, é provável que a redução no número de amostras com sementes tenha ocorrido em função do maior grau de agregação da chuva de sementes, corroborando o padrão espacial de sementes e plântulas que vem sendo descrito para áreas com densa cobertura de bambus.

Considerando que $A$. aristulata é uma espécie de bambu lenhoso que, assim como outras espécies de bambus, apresenta crescimento clonal, vigorosa produção de biomassa (Narukawa \& Yamamoto 2002) e aparentemente mantém o estado de perturbação da floresta, sua influência sobre a estrutura da comunidade pode ter um efeito negativo sobre a disponibilidade de sementes no banco transitório. Em nosso estudo, não encontramos diferenças entre as áreas em relação à abundância de sementes do banco. Entretanto, estudos realizados no local mostraram que a chuva de sementes foi cerca de seis vezes mais abundante na área $\mathrm{CB}$ em comparação com a SB (Grombone-Guaratini et al. 2014). Diante dessa condição, seria esperado que o banco transitório da área com bambu tendesse a refletir a abundância de sementes dispersas na chuva de sementes. Como consequência, nossos resultados sugerem que há uma substancial perda de sementes no banco transitório da área $\mathrm{CB}$, o que poderia estar relacionada com uma maior susceptibilidade à predação. Estudos realizados em regiões tropicais têm mostrado que o aumento na densidade de bambus tem um efeito positivo sobre a retenção de sementes ao redor das moitas. No entanto, esse efeito é direcional, uma vez que há uma redução na chance de retenção de sementes de maior tamanho e massa (sementes típicas do banco transitório) devido ao seu valor energético e conteúdo nutricional atraírem predadores, o que poderia reduzir as fontes de sementes para a regeneração desse grupo (Qian et al. 2016).

Muitos estudos têm avaliado o banco de sementes do solo em áreas perturbadas, contudo, o banco de sementes transitório raramente tem sido investigado. Até onde temos conhecimento, este é o primeiro estudo a descrever a estrutura e a composição do banco de sementes transitório em áreas com dominância por bambus. Nosso estudo demonstrou que a análise do conteúdo de sementes contidas na serapilheira se mostra um método eficaz para o levantamento de informações diagnósticas do estado de perturbação florestal. Este estudo mostrou que o banco de sementes transitório tem baixo potencial para contribuir para a regeneração natural em situações em que ocorre superabundância por bambus. Embora a abundância de sementes possa apresentar-se aparentemente inalterada, a redução na riqueza de espécies, o aumento da agregação espacial das sementes e alterações na composição florística desse componente da regeneração podem contribuir para a perpetuação do bambu e para a manutenção do estado de distúrbio. Desse modo, técnicas de manejo que consideram a remoção parcial do bambu, como propostas por Campanello et al. (2007), podem ser insuficientes na indução da regeneração natural por meio do banco de sementes transitório, a menos que outras técnicas, como o enriquecimento com sementes e mudas de espécies nativas possam ser utilizadas de modo complementar.

\section{Material Suplementar}

Este artigo possui material suplementar em formato digital que contêm os dados coletados e utilizados nas análises, correspondendo a: 1) abundância de sementes no banco de sementes transitório; 2) lista de espécies do banco de sementes transitório; e 3) matriz de riqueza de espécies. Os dados podem ser acessados por meio do link: $<$ https:/figshare.com/s/b10a682c1d6a07666fc9>.

\section{Agradecimentos}

A primeira autora agradece ao $\mathrm{CNPq}$ (Conselho Nacional de Desenvolvimento Científico e Tecnológico), a bolsa de Mestrado concedida, e ao Programa de Pós-graduação em Biodiversidade Vegetal e Meio Ambiente do Instituto de Botânica 
de São Paulo. Nossos sinceros agradecimentos à Dra. Inês Cordeiro, ao Dr. Eduardo L. Catharino, à Dra. Fátima O. de Souza, e ao Sr. Francismar F.A. Aguiar, o auxílio na identificação das sementes; ao Dr. André Rochelle, por nos auxiliar com o georreferenciamento das parcelas, e ao Sr. Carlos Augusto Giordano Bata de Oliveira, o tratamento das figuras. Somos gratas também aos dois revisores anônimos, bem como ao editor de área, que contribuíram valiosamente com suas avaliações e sugestões críticas. Esta pesquisa recebeu auxílio financeiro da Fundação de Amparo à Pesquisa do estado de São Paulo (FAPESP 05/51747-2).

\section{Referências}

Alvarez-Aquino C, Williams-Linera G \& Newton AC (2005) Disturbance effects on the seed bank of Mexican cloud forest fragments. Biotropica 37: 337-432.

Alvarez-Buylla ER \& García-Barrios R (1991) Seed and forest dynamics: a theoretical framework and an example from the neotropics. The American Naturalist 137: 133-154.

APG IV - Angiosperm Phylogeny Group (2016) An update of the Angiosperm Phylogeny Group classification for the orders and families of flowering plants. Botanical Journal of the Linnean Society 181: 1-20.

Barros F, Mamede MCH, Melo MMRF, Lopes E, JungMendaçolli S, Kirizawa M, Muniz CFS, MakinoWatanabe H, Chiea SAC \& Melhem TS (2002) A Flora Fanerogâmica do PEFI: composição, afinidades e conservação. In: Bicudo DC, Forti MC \& Bicudo CEM (eds.) Parque Estadual das Fontes do Ipiranga: uma reserva Biológica na cidade de São Paulo, São Paulo. Secretaria do Meio Ambiente do estado de São Paulo, São Paulo. Pp. 93-110.

Campanello PI, Gatti MG, Ares A, Montti L \& Goldstein G (2007) Tree regeneration and microclimate in a liana and bamboo-dominated semideciduous Atlantic Forest. Forest Ecology and Management 252: 108-117.

Castillo LS \& Stevenson PR (2010) Relative importance of seed-bank and post-disturbance seed dispersal on early gap. Biotropica 42: 488-492.

Clark LG (2001) Aulonemia. In: Wanderley MGL, Shepherd GJ, Giulietti AM \& Melhem TS (eds.) Flora Fanerogâmica do estado de São Paulo. Instituto de Botânica, São Paulo. Vol. 1, pp. 23-24.

Dalling JW \& Hubbell SP (2002) Seed size, growth rate and gap microsite conditions as determinants of recruitment success for pioneer species. Journal of Ecology 90: 557-568.

d'Oliveira MV, Guarino ES, Oliveira L, Ribas LA \& Acuña MHA (2013) Can forest management be sustainable in a bamboo dominated forest? A 12-year study of forest dynamics in western Amazon. Forest Ecology and Management 310: 672-679.
EMBRAPA - Centro Natural de Pesquisa de Solos (2006) Sistema brasileiro de classificação de solos, Rio de Janeiro. 306p.

Farrell C, Hobbs RJ \& Colmer TD (2012) Microsite and litter cover effects on seed banks vary with seed size and dispersal mechanisms: implications for revegetation of degraded saline land. Plant Ecology 213: 1145-1155.

Filgueiras TS \& Pereira BAS (1988) On the flowering of Actinocladum verticillatum (Gramineae: Bambusoideae). Biotropica 20: 164-166.

Filgueiras TS \& Santos-Gonçalves AP (2004) A checklist of the basal grasses and bamboos in Brazil (Poaceae). Bamboo Science and Culture 18: 7-18.

Foster SA (1986) On the adaptive value of large seeds for tropical moist forest trees: a review and synthesis. The Botanical Review 52: 260-299.

Garrot RA, White PJ \& White CAV (1993) Overabundance: an issue for conservation biologists? Conservation Biology 7: 946-949.

Garwood NC (1989) Tropical soil seed banks: a review. In: Leck MA, Parker VT \& Simpson RL (eds.) Ecology of soil seed banks. Academic Press, New York. Pp. 149-205.

González ME, Veblen TT, Donoso C \& Valeria L (2002) Tree regeneration responses in a lowland Notophagusdominated forest after bamboo dieback in SouthCentral Chile. Plant Ecology 161: 59-73.

Gotelli NJ \& Colwell RK (2001) Quantifying biodiversity: procedures and pitfalls in the measurement and comparison of species richness. Ecological Letters4: 79-391.

Gotelli NJ \& Entsminger GL (2001) EcoSim: Null models software for ecology. Version 7.0. Acquired Intelligence Inc. \& Kesey-Bear. Disponível em <http://www.uvm. edu/ ngotelli/EcoSim/EcoSim.html $>$. Acesso em 15 março 2015.

Gratani L, Crescente MF, Varone L, Fabrini G \& Digiulio E (2008) Growth pattern and photosynthetic activity of different bamboo species growing in the Botanical Garden of Rome. Flora 203: 77-84.

Griscom BW \& Ashton PMS (2003) Bamboo control of forest succession: Guadua sarcocarpa in southeastern Peru. Forest Ecology and Management 175: 445-454.

Griscom BW \& Ashton PMS (2006) A self-perpetuating bamboo disturbance cycle in a neotropical forest. Journal of Tropical Ecology 22: 587-597.

Griscon BW, Daly DC \& Ashton MS (2007) Floristics of bamboo-dominated stands in lowland terra-firma forests of southwestern Amazonia. Journal of Torrey Botanical Society 134: 108-125.

Grombone-Guaratini MT, Alves LF, Vinha D \& Franco GADC (2014) Seed rain in areas with and without bamboo dominance within an urban fragment of the Atlantic forest. Acta Botanica Brasilica 28: 76-85.

Köppen W (1948) Climatologia: com um estudio de los climas de la tierra. Fondo de Cultura Económica, México. 478p. 
Larpken P, Moe SR \& Totland Ǿ (2011) Bamboo dominance reduces tree regeneration in a disturbed tropical forest. Oecologia 165: 161-168.

Lima RAF, Rother DC, Muller AE, Lepsch I \& Rodrigues RR (2012) Bamboo overabundance alters forest structure and dynamics in the Atlantic Forest hotspot. Biological Conservation 147: 32-39.

Magurran AE \& Mcgill BJ (2011) Biological diversity: frontiers in measurement and assessment. Oxford University Press, New York. 345p.

Mônico AC (2012) Transferência do banco de sementes superficiais como estratégia de enriquecimento em uma floresta em processo de regeneração. Dissertação de Mestrado. Universidade de São Paulo, Piracicaba. $174 \mathrm{p}$.

Montti L, Villagra M, Campanello PI, Gatti MG \& Goldstein G (2013) Functional traits enhance invasiveness of bamboo over co-occurring tree saplings in the semideciduous Atlantic Forest. Acta Oecologica 37: 361-368.

Moro MF, Souza VC, Oliveira-Filho AT, Queiroz LP, Fraga CN, Rodal MJN, Araújo FS \& Martins FR (2012) Alienígenas na sala: o que fazer com espécies exóticas em trabalhos de taxonomia, florística e fitossociologia? Acta Botanica Brasilica 26: 991-999.

Müeller-Dombois D \& Ellenberg H (1974) Aims and methods of vegetation ecology. John Wiley, New York. 547p.

Mulkey SS (1986) Photosynthetic acclimation and water-use efficiency of three species of understory herbaceous bamboo (Gramineae) in Panama. Oecologia 70: 514-519.

Narukawa Y \& Yamamoto S (2002) Effects of dwarf bamboo (Sasa sp.) and forest floor microsites on conifer seedling recruitment in a subalpine forest, Japan. Forest Ecology and Management 163: 61-70.

Nastri VDF, Catharino ELM, Rossi L, Barbosa LM, Pirré E, Bedinelli C, Asperti LM, Dorta RO \& Costa MP(1992) Estudos fitossociológicos de uma área do Instituto de Botânica de São Paulo utilizada em programa de educação ambiental. Revista do Instituto Florestal 4: 219-225.

Oliveira-Filho AT \& Fontes MA (2000) Patterns of floristic differentiation among Atlantic forests in southeastern Brazil and the influence of climate. Biotropica 32: 793-810.

Omand KA, Karberg JM, Beattie KC, O'Dell DI \& Freeman RS (2014) Soil seed bank in nantucket's early successional communities: implications for management. Natural Areas Journal 34: 188-198.

Penhalber EF \& Mantovani W (1997) Floração e chuva de sementes em mata secundária em São Paulo, SP. Revista Brasileira de Botânica 20: 205-220.

Pivello VR \& Peccinini AA (2002) A vegetação do PEFI. In: Bicudo DC, Forti MC \& Bicudo CEM (eds.) Parque Estadual das Fontes do Ipiranga: uma reserva Biológica na cidade de São Paulo, São Paulo. Secretaria do Meio Ambiente do estado de São Paulo, São Paulo. Pp. 75-92.

Qian F, Zhang T, Guo Q \& Tao J (2016) Dense understory dwarf bamboo alters the retention of canopy tree seeds. Acta Oecologica 73: 38-44.

Rodrigues BD, Martins SV \& Leite HG (2010)Avaliação do potencial de transposição da serapilheira e do banco de sementes do solo para a restauração florestal em áreas degradadas. Revista Árvore 34: 65-73.

Rother DC, Rodrigues RR \& Pizo MA (2009) Effects of bamboo stands on seed rain and seed limitation in a rainforest. Forest Ecology and Management 257: 885-892.

Rother DC, Pizo MA, Siqueira T, Rodrigues RR \& Jordano $P$ (2015) Community-wide spatial and temporal discordance of seed-seedling shadows in a tropical rainforest. PlosOne 10: e0123346.

Rother DC, Rodrigues RR \& Pizo MA (2016) Bamboo thickets alter the demographic structure of Euterpe edulis population: A keystone, threatened palm species of the Atlantic forest. Acta Oecologica 70: 96-102.

Santos PM \& Funari FL (2002) Clima Local. In: Bicudo DC, Forti MC \& Bicudo CEM (eds.) Parque Estadual das Fontes do Ipiranga: uma reserva Biológica na cidade de São Paulo, São Paulo. Secretaria do Meio Ambiente do estado de São Paulo, São Paulo. Pp. 29-48.

Shackelford NRJ, Hobbs RJ, Heller NE, Hallett LM \& Seasted LM (2013) Finding a middle-ground: The native/non-native debate. Biological Conservation 158: 55-62.

Shirasuna RT \& Filgueiras TS (2013) Bambus nativos (Poaceae, Bambusoideae) no Parque Estadual das Fontes do Ipiranga, São Paulo, SP, Brasil. Hoehnea 40: 315-359.

Simpson RL, Leck MA \& Parker VT (1989) Seed banks: general concepts and methodological issues. In: Leck MA, Parker VT \& Simpson RL (eds.) Ecology of soil seed banks. Academic Press, London. 462p.

Souza PA, Venturin N, Griffith JJ \& Martins SV (2006) Avaliação do banco de sementes contido na serapilheira de um fragmento florestal visando recuperação de áreas degradadas. Cerne 12: 56-67.

Tabarelli M \& Mantovani W (1999) Clareiras naturais e a riqueza de espécies pioneiras em uma floresta Atlântica montana. Revista Brasileira de Biologia 59: 251-261.

Vinha D, Alves LF, Zaidan LBP \& Grombone-Guaratini MT (2011) The potential of the soil seed bank for the regeneration of a tropical urban forest dominated by bamboo. Landscape and Urban Planning 99: 178-185.

Widmer Y (1998) Pattern and performance of understorey bamboos (Chusquea spp) under different canopy closures in old-growth oak forests in Costa Rica. Biotropica 30: 400-415.

Zar JH (2010) Biostatistical analysis. Prentice Hall, New Jersey. 944p. 